\title{
Convergence rates of derivatives of a family of barycentric rational interpolants*
}

\author{
Jean-Paul Berrut ${ }^{\dagger} \quad$ Michael S. Floater ${ }^{\ddagger} \quad$ Georges Klein $^{\dagger}$
}

\begin{abstract}
In polynomial and spline interpolation the $k$-th derivative of the interpolant, as a function of the mesh size $h$, typically converges at the rate of $O\left(h^{d+1-k}\right)$ as $h \rightarrow 0$, where $d$ is the degree of the polynomial or spline. In this paper we establish, in the important cases $k=1,2$, the same convergence rate for a recently proposed family of barycentric rational interpolants based on blending polynomial interpolants of degree $d$.
\end{abstract}

Math Subject Classification: 65D05, 41A05, 41A20, 41A25

Keywords: Rational interpolation, barycentric form, convergence rate.

\section{Introduction}

In a recent paper [7], a family of barycentric rational interpolants was investigated. Given a function $f:[a, b] \rightarrow \mathbb{R}$ and real values ("nodes") $a=x_{0}<x_{1}<\cdots<x_{n}=b$, the $d$-th interpolant, $d=0,1, \ldots, n$, is given by the formula

$$
r(x)=\sum_{i=0}^{n-d} \lambda_{i}(x) p_{i}(x) / \sum_{i=0}^{n-d} \lambda_{i}(x),
$$

where $p_{i}$ denotes the polynomial of degree $\leq d$ such that $p_{i}\left(x_{j}\right)=f\left(x_{j}\right)$ for $j=i, i+$ $1, \ldots, i+d$, and

$$
\lambda_{i}(x)=\frac{(-1)^{i}}{\left(x-x_{i}\right)\left(x-x_{i+1}\right) \cdots\left(x-x_{i+d}\right)}
$$

is a blending function. It was shown in [7] that for $f \in C^{d+2}[a, b]$, the error,

$$
e(x):=f(x)-r(x),
$$

\footnotetext{
*Work partly supported by the Swiss National Science Foundation under grant No. 200020-124779.

${ }^{\dagger}$ Department of Mathematics, University of Fribourg, Pérolles, 1700 Fribourg, Switzerland, email: \{jean-paul.berrut,georges.klein\}@unifr.ch

${ }_{\ddagger}^{\ddagger}$ Centre of Mathematics for Applications, Department of Informatics, University of Oslo, PO Box 1053 Blindern, 0316 Oslo, Norway, email: michaelf@ifi.uio.no
} 
satisfies a bound on the max norm $\|e\|:=\max _{a \leq x \leq b}|e(x)|$ of the form

$$
\|e\| \leq C h^{d+1}
$$

where $h$ is the mesh size,

$$
h:=\max _{0 \leq i \leq n-1}\left(x_{i+1}-x_{i}\right) .
$$

Here and in all what follows, $C$ is a constant depending only on $d$, on derivatives of $f$, and on the interval length $b-a$. Thus, for smooth enough $f$, the interpolant $r$ converges to $f$ at the rate $O\left(h^{d+1}\right)$ as $h \rightarrow 0$.

A natural, though not definitely settled question is that of a good or even optimal choice of the parameter $d$ for a given function. Through the estimate (3) one might be tempted to think that $d$ should be taken very large; however, the fact that the $p_{i}(x)$ in $(1)$ are polynomials of degree up to $d$, interpolating between arbitrary nodes, necessarily restricts $d$ (due to Runge's phenomenon and instability). Nevertheless, numerical experience shows that $d$ may often be chosen quite large. The authors of [10] remark: "This is the only method in this chapter for which we might actually encourage experimentation with high order (say, $>6$ )". Furthermore, as this family of rational interpolants is linear in the data, the condition of $r$ is determined by its Lebesgue constant $\Lambda_{n}$ [4]. Several authors have recently studied $\Lambda_{n}$ and shown that it may be bounded for equidistant nodes and $d \geq 2$ as $[5]$

$$
\frac{2^{d-2}}{d+1} \ln (n / d-1) \leq \Lambda_{n} \leq 2^{d-1}(2+\ln (n))
$$

thereby confirming that $d$ may be increased to values which yield a very favourable error decay with $n$ (up to about $d=20$ for large values of $n$ in some cases).

With a view to possible applications such as the numerical solution of differential equations [2], we study here the rate of convergence of derivatives of $r$ to corresponding derivatives of $f$. Taking into account that $r$ is a blend of polynomial interpolants of degree at most $d$, it is not unreasonable to expect that

$$
\left\|e^{(k)}\right\| \leq C h^{d+1-k}
$$

for $k=1,2, \ldots, d$. In this paper we prove that this holds for $k=1$ and $k=2$, in some cases under addition of a mesh ratio condition on the nodes $x_{i}$; such a condition holds, for example, in the important equally spaced case, $x_{i}=a+i(b-a) / n$. We conjecture that (4) is valid, at least in the equally spaced case, for all $k=1,2, \ldots, d$. No fundamental reason hinders the extension of the method to the cases $k \geq 3$; the difficulty is that the formulas for $e^{(k)}$ become very intricate. In Section 2 we will look more closely at the rate of convergence at the nodes, while in Section 3 we extend the theory to intermediate points. The paper ends with numerical examples which confirm the mathematical analysis.

\section{Error at the nodes}

The Newton error formula

$$
f(x)-p_{i}(x)=\left(x-x_{i}\right) \cdots\left(x-x_{i+d}\right)\left[x_{i}, x_{i+1}, \ldots, x_{i+d}, x\right] f
$$


leads to

$$
e(x)=\frac{\sum_{i=0}^{n-d} \lambda_{i}(x)\left(f(x)-p_{i}(x)\right)}{\sum_{i=0}^{n-d} \lambda_{i}(x)}=\frac{A(x)}{B(x)},
$$

where after cancellation

$$
A(x):=\sum_{i=0}^{n-d}(-1)^{i}\left[x_{i}, \ldots, x_{i+d}, x\right] f
$$

and

$$
B(x):=\sum_{i=0}^{n-d} \lambda_{i}(x) .
$$

Consider the first derivative of $e$ at a node $x_{j}, 0 \leq j \leq n$. By the definition of the derivative and the fact that $e\left(x_{j}\right)=0$, we have

$$
e^{\prime}\left(x_{j}\right)=\lim _{x \rightarrow x_{j}} \frac{e(x)}{x-x_{j}} .
$$

This motivates us to look at the product $\left(x-x_{j}\right) B(x)$. Defining the functions

$$
B_{j}(x):=\sum_{i \in I_{j}}(-1)^{i} \prod_{\substack{k=i \\ k \neq j}}^{i+d} \frac{1}{x-x_{k}}
$$

and

$$
C_{j}(x):=\sum_{i \in I \backslash I_{j}}(-1)^{i} \prod_{k=i}^{i+d} \frac{1}{x-x_{k}},
$$

where

$$
I=\{0,1, \ldots, n-d\}
$$

and

$$
I_{j}=\{i \in I: j-d \leq i \leq j\},
$$

we see that

$$
\left(x-x_{j}\right) B(x)=B_{j}(x)+\left(x-x_{j}\right) C_{j}(x) .
$$

Lemma 1

$$
e^{\prime}\left(x_{j}\right)=\frac{A\left(x_{j}\right)}{B_{j}\left(x_{j}\right)}
$$

Proof. By (10) we have

$$
\frac{e(x)}{x-x_{j}}=\frac{A(x)}{B_{j}(x)+\left(x-x_{j}\right) C_{j}(x)},
$$

and taking the limit of both sides as $x \rightarrow x_{j}$ gives the result. 
We can use this formula to obtain an error bound at the nodes that requires $f$ to be in $C^{d+2}[a, b]$, the same as for the bound (3). To increase readability, we introduce the following notation:

$$
d_{i}(x):=\left|x-x_{i}\right|, \quad d_{i k}:=\left|x_{i}-x_{k}\right|,
$$

for nodes $x_{i}$ and $x_{k}$ and for $x \in[a, b]$, and when it is clear, we also write $d_{i}=d_{i}(x)$.

Theorem 1 If $f \in C^{d+2}[a, b]$, then

$$
\left|e^{\prime}\left(x_{j}\right)\right| \leq C h^{d}, \quad 0 \leq j \leq n .
$$

Proof. With $x=x_{j}$ in (8), the products alternate in sign as $(-1)^{i}$ does, so that all the terms in the sum have the same sign and

$$
\left|B_{j}\left(x_{j}\right)\right|=\sum_{i \in I_{j}} \prod_{\substack{k=i \\ k \neq j}}^{i+d} d_{j k}^{-1}
$$

Therefore, by choosing any $i \in I_{j}$, we deduce that

$$
\frac{1}{\left|B_{j}\left(x_{j}\right)\right|} \leq \prod_{\substack{k=i \\ k \neq j}}^{i+d} d_{j k} \leq C h^{d}, \quad \forall i \in I_{j} .
$$

On the other hand it has been shown in [7] that

$$
|A(x)| \leq C, \quad x \in[a, b],
$$

whence the bound follows.

To deal with higher derivatives, we consider the Taylor expansion

$$
e(x)=\left(x-x_{j}\right) e^{\prime}\left(x_{j}\right)+\frac{1}{2 !}\left(x-x_{j}\right)^{2} e^{\prime \prime}\left(x_{j}\right)+\frac{1}{3 !}\left(x-x_{j}\right)^{3} e^{\prime \prime \prime}\left(x_{j}\right)+\cdots
$$

and the Taylor expansion of

$$
q_{j}(x):=\frac{e(x)}{x-x_{j}}
$$

namely

$$
q_{j}(x)=q_{j}\left(x_{j}\right)+\left(x-x_{j}\right) q_{j}^{\prime}\left(x_{j}\right)+\frac{1}{2 !}\left(x-x_{j}\right)^{2} q_{j}^{\prime \prime}\left(x_{j}\right)+\cdots .
$$

Dividing (14) by $x-x_{j}$ and comparing terms in the two expansions imply that

$$
e^{(k)}\left(x_{j}\right)=k q_{j}^{(k-1)}\left(x_{j}\right),
$$

in particular,

$$
e^{\prime \prime}\left(x_{j}\right)=2 q_{j}^{\prime}\left(x_{j}\right)
$$


Differentiating (11) and substituting $x=x_{j}$ give

$$
q_{j}^{\prime}\left(x_{j}\right)=\frac{A^{\prime}\left(x_{j}\right)}{B_{j}\left(x_{j}\right)}-\frac{B_{j}^{\prime}\left(x_{j}\right) A\left(x_{j}\right)}{B_{j}^{2}\left(x_{j}\right)}-\frac{C_{j}\left(x_{j}\right) A\left(x_{j}\right)}{B_{j}^{2}\left(x_{j}\right)},
$$

which we will use to derive a bound for $e^{\prime \prime}\left(x_{j}\right)$. We begin with a lemma.

Lemma 2 If $f \in C^{d+2+k}[a, b]$ for $k \in \mathbb{N}$, then

$$
\left|A^{(k)}(x)\right| \leq C, \quad x \in[a, b] .
$$

Proof. The case $k=0$ has been treated in [7]. For $k \neq 0$ and using the derivative formula for divided differences (see [1] and [8]), we have

$$
A^{(k)}(x)=k ! \sum_{i=0}^{n-d}(-1)^{i}\left[x_{i}, \ldots, x_{i+d},(x)^{k+1}\right] f,
$$

where $(x)^{k}$ stands for a $k$-fold argument. Then, with a similar approach to [7, p.322], $A^{(k)}(x) / k$ ! equals

$$
\begin{aligned}
& -\sum_{i=0, i \text { even }}^{n-d-1}\left(x_{i+d+1}-x_{i}\right)\left[x_{i}, \ldots, x_{i+d+1},(x)^{k+1}\right] f, \quad(n-d) \text { odd, } \\
& -\sum_{i=0, i \text { even }}^{n-d-2}\left(x_{i+d+1}-x_{i}\right)\left[x_{i}, \ldots, x_{i+d+1},(x)^{k+1}\right] f+\left[x_{n-d}, \ldots, x_{n},(x)^{k+1}\right] f, \quad(n-d) \text { even. }
\end{aligned}
$$

Using the same argument as in [7], we are done.

Theorem 2 If $d \geq 1$ and if $f \in C^{d+3}[a, b]$, then

$$
\left|e^{\prime \prime}\left(x_{j}\right)\right| \leq C h^{d-1}
$$

Proof. We write equation (16) with (17) in the form

$$
e^{\prime \prime}\left(x_{j}\right)=2\left(L_{1}-L_{2}-L_{3}\right),
$$

where

$$
L_{1}:=\frac{A^{\prime}\left(x_{j}\right)}{B_{j}\left(x_{j}\right)}, \quad L_{2}:=\frac{B_{j}^{\prime}\left(x_{j}\right) A\left(x_{j}\right)}{B_{j}^{2}\left(x_{j}\right)}, \quad L_{3}:=\frac{C_{j}\left(x_{j}\right) A\left(x_{j}\right)}{B_{j}^{2}\left(x_{j}\right)}
$$

and we show that

$$
\left|L_{1}\right| \leq C h^{d}
$$

and

$$
\left|L_{2}\right|,\left|L_{3}\right| \leq C h^{d-1}
$$


Equation (18) immediately follows from equation (12) and Lemma 2. To deal with $L_{2}$, we notice that

$$
B_{j}^{\prime}(x)=\sum_{i \in I_{j}}(-1)^{i+1} \sum_{\substack{m=i \\ m \neq j}}^{i+d} \frac{1}{x-x_{m}} \prod_{\substack{k=i \\ k \neq j}}^{i+d} \frac{1}{x-x_{k}}
$$

so that its absolute value in $x=x_{j}$ is bounded by

$$
\left|B_{j}^{\prime}\left(x_{j}\right)\right| \leq \sum_{i \in I_{j}} \sum_{\substack{m=i \\ m \neq j}}^{i+d} d_{j m}^{-1} \prod_{\substack{k=i \\ k \neq j}}^{i+d} d_{j k}^{-1}
$$

To derive a bound of the quotient of $(20)$ with $\left|B_{j}\left(x_{j}\right)\right|^{2}$, we use (12) with $i \in I_{j}$ equal to the index in the outer sum in $(20)$ :

$$
\frac{\left|B_{j}^{\prime}\left(x_{j}\right)\right|}{\left|B_{j}\left(x_{j}\right)\right|^{2}} \leq \sum_{i \in I_{j}} \sum_{\substack{m=i \\ m \neq j}}^{i+d} d_{j m}^{-1} \prod_{\substack{k=i \\ k \neq j}}^{i+d} d_{j k}^{2} \prod_{\substack{k=i \\ k \neq j}}^{i+d} d_{j k}^{-1} \leq \sum_{i \in I_{j}} \sum_{\substack{m=i \\ m \neq j}}^{i+d} \prod_{\substack{k=i \\ k \neq j \\ k \neq m}}^{i+d} d_{j k} \leq C h^{d-1}
$$

and this, together with (13), gives the bound on $L_{2}$ in (19).

Finally, we treat $L_{3}$. We split $C_{j}\left(x_{j}\right)$ into two parts,

$$
C_{j}\left(x_{j}\right)=\sum_{i=0}^{j-d-1}(-1)^{i} \prod_{k=i}^{i+d} \frac{1}{x_{j}-x_{k}}+\sum_{i=j+1}^{n-d}(-1)^{i} \prod_{k=i}^{i+d} \frac{1}{x_{j}-x_{k}},
$$

where empty sums are meant to equal 0 . The terms in both sums are alternating in sign and increasing, respectively decreasing, in absolute value, so that

$$
\left|C_{j}\left(x_{j}\right)\right| \leq \prod_{k=j-d-1}^{j-1} d_{j k}^{-1}+\prod_{k=j+1}^{j+1+d} d_{j k}^{-1} .
$$

We now divide every term in equation $(21)$ by $\left|B_{j}\left(x_{j}\right)\right|^{2}$. Using (12) with $i=j-d$ for the first term and $i=j$ for the second, we obtain

$$
\frac{\left|C_{j}\left(x_{j}\right)\right|}{\left|B_{j}\left(x_{j}\right)\right|^{2}} \leq \frac{\prod_{k=j-d}^{j-1} d_{j k}^{2}}{\prod_{k=j-d-1}^{j-1} d_{j k}}+\frac{\prod_{k=j+1}^{j+d} d_{j k}^{2}}{\prod_{k=j+1}^{j+d+1} d_{j k}}=\frac{\prod_{k=j-d}^{j-1} d_{j k}}{d_{j, j-d-1}}+\frac{\prod_{k=j+1}^{j+d} d_{j k}}{d_{j, j+d+1}} .
$$

Since

it follows that

$$
\frac{d_{j, j-d}}{d_{j, j-d-1}} \leq 1, \quad \frac{d_{j, j+d}}{d_{j, j+d+1}} \leq 1
$$

$$
\frac{\left|C_{j}\left(x_{j}\right)\right|}{\left|B_{j}\left(x_{j}\right)\right|^{2}} \leq \prod_{k=j-d+1}^{j-1} d_{j k}+\prod_{k=j+1}^{j+d-1} d_{j k} \leq C h^{d-1},
$$

which, together with (13), gives the bound on $L_{3}$ in (19). 


\section{Error at intermediate points}

We now consider the rate of convergence of the derivative of the error at any point $x$ in $[a, b]$. For the first derivative we obtain the same rate of convergence as at the nodes, namely $O\left(h^{d}\right)$, but only under the stricter condition that $f \in C^{d+3}[a, b]$.

Theorem 3 If $d \geq 2$ and if $f \in C^{d+3}[a, b]$, then

$$
\left\|e^{\prime}\right\| \leq C h^{d} .
$$

Proof. Due to the continuity of $e^{\prime}$, it is sufficient to let $x \in\left(x_{j}, x_{j+1}\right)$ and to show that

$$
\left|e^{\prime}(x)\right| \leq C h^{d}
$$

independently of $j$. To establish (22), we differentiate (5), to obtain

$$
e^{\prime}(x)=\frac{A^{\prime}(x)}{B(x)}-A(x) \frac{B^{\prime}(x)}{B^{2}(x)} .
$$

In the proof of Theorem 2 of [7] it was shown that

$$
|B(x)| \geq \frac{1}{C h^{d+1}}, \quad \forall x \in[a, b]
$$

and so, from Lemma 2, it follows that

$$
\frac{\left|A^{\prime}(x)\right|}{|B(x)|} \leq C h^{d+1}
$$

Since $|A(x)| \leq C$, it remains to show that

$$
\frac{\left|B^{\prime}(x)\right|}{\left|B^{2}(x)\right|} \leq C h^{d}
$$

We use the following index sets introduced in [7] and which subdivide $I$ :

$J_{1}=\{i \in I: i \leq j-d\}, \quad J_{2}=\{i \in I: j-d+1 \leq i \leq j\}, \quad J_{3}=\{i \in I: j+1 \leq n-d\}$.

Now

$$
\left|B^{\prime}(x)\right|=\left|\sum_{i \in I} \sum_{m=0}^{d} \frac{(-1)^{i+1}}{\left(x-x_{i}\right) \cdots\left(x-x_{i+d}\right)\left(x-x_{i+m}\right)}\right| \leq \sum_{m=0}^{d}\left(M_{m, 1}+M_{m, 2}+M_{m, 3}\right),
$$

where we have interchanged the summation order and set

$$
M_{m, p}:=\left|\sum_{i \in J_{p}} \frac{(-1)^{i+1}}{\left(x-x_{i}\right) \cdots\left(x-x_{i+d}\right)\left(x-x_{i+m}\right)}\right|, \quad p=1,2,3 .
$$


If $J_{p}=\varnothing$, then $M_{m, p}=0 . J_{2}$ is not empty since $d \geq 2$.

For every fixed $m$, the terms in the sums in $M_{m, 1}$ and $M_{m, 3}$ are alternating in sign and increasing, respectively decreasing, in absolute value and so

$$
M_{m, 1} \leq \frac{1}{d_{j-d} \cdots d_{j} d_{j-d+m}} \quad \text { and } \quad M_{m, 3} \leq \frac{1}{d_{j+1} \cdots d_{j+1+d} d_{j+1+m}}
$$

In the same proof in [7, p. 322], it has been shown that

$$
|B(x)| \geq\left|\lambda_{i}(x)\right|, \quad \forall i \in J_{2} .
$$

Next, we divide $M_{m, 1}$ by $|B(x)|^{2}$ and use (26) with $i=j-d+1$, so that

$$
\frac{M_{m, 1}}{|B(x)|^{2}} \leq \frac{d_{j-d+1}^{2} \cdots d_{j+1}^{2}}{d_{j-d} \cdots d_{j} d_{j-d+m}}=\frac{d_{j-d+1} \cdots d_{j} d_{j+1}^{2}}{d_{j-d} d_{j-d+m}}
$$

and since $d_{j} / d_{j-d+m} \leq 1$ for $m=0, \ldots, d$ and $d_{j-d+1} / d_{j-d} \leq 1$ :

$$
\frac{M_{m, 1}}{|B(x)|^{2}} \leq d_{j-d+2} \cdots d_{j-1} d_{j+1}^{2} \leq C h^{d}
$$

Similarly

$$
\frac{M_{m, 3}}{|B(x)|^{2}} \leq C h^{d}
$$

Finally we bound $M_{m, 2} /|B(x)|^{2}$. Choosing the same $i \in J_{2}$ in (26) as in each term of the sum in $M_{m, 2}$, it follows

$$
\frac{M_{m, 2}}{|B(x)|^{2}} \leq \sum_{i \in I_{2}} \frac{d_{i}^{2} \cdots d_{i+d}^{2}}{d_{i} \cdots d_{i+d} d_{i+m}}=\sum_{i \in I_{2}} d_{i} \cdots d_{i+m-1} d_{i+m+1} \cdots d_{i+d} \leq C h^{d} .
$$

Thus (24) follows from (25).

In the case $d=1$, we obtain the same rate of convergence, $O(h)$, as for the larger $d$ in Theorem 3 but only under a bounded mesh ratio.

Theorem 4 If $d=1$ and if $f \in C^{4}[a, b]$, then

$$
\left\|e^{\prime}\right\| \leq C(2 \beta+1) h,
$$

where

$$
\beta:=\max \left\{\max _{1 \leq i \leq n-1} \frac{d_{i, i+1}}{d_{i, i-1}}, \max _{0 \leq i \leq n-2} \frac{d_{i+1, i}}{d_{i+1, i+2}}\right\} .
$$


Proof. Again we determine the open subinterval $\left(x_{j}, x_{j+1}\right)$ containing $x$ and consider (23). Since the bounds for $|A(x)|,\left|A^{\prime}(x)\right|$ and $|B(x)|$ from the previous theorem also hold for $d=1$, we bound $\left|B^{\prime}(x)\right| /|B(x)|^{2}$ for $d=1$ and $J_{2}=\{j\}$. Using similar arguments as in that theorem, we obtain

$$
\begin{aligned}
\frac{\left|B^{\prime}(x)\right|}{|B(x)|^{2}} & \leq \sum_{m=0}^{1}\left(\frac{1}{|B(x)|^{2}}\left|\sum_{i \in I} \frac{(-1)^{i+1}}{\left(x-x_{i}\right)\left(x-x_{i+1}\right)\left(x-x_{i+m}\right)}\right|\right) \\
& \leq \sum_{m=0}^{1}\left(\frac{d_{j}^{2} d_{j+1}^{2}}{d_{j-1} d_{j} d_{j-1+m}}+\frac{d_{j}^{2} d_{j+1}^{2}}{d_{j} d_{j+1} d_{j+m}}+\frac{d_{j}^{2} d_{j+1}^{2}}{d_{j+1} d_{j+2} d_{j+1+m}}\right) \\
& \leq 2 \frac{d_{j+1}^{2}}{d_{j-1}}+d_{j+1}+d_{j}+2 \frac{d_{j}^{2}}{d_{j+2}} \\
& \leq 2(2 \beta+1) h .
\end{aligned}
$$

For the second derivative the mesh ratio enters every bound.

Theorem 5 If $d \geq 3$ and if $f \in C^{d+4}[a, b]$, then

$$
\left\|e^{\prime \prime}\right\| \leq C(\beta+1) h^{d-1} .
$$

Proof. We continue to work with $x \in\left(x_{j}, x_{j+1}\right)$, and we express the error $e$ in (5) as

$$
e(x)=\psi(x) \widetilde{e}(x)
$$

where

$$
\psi(x):=\left(x-x_{j}\right)\left(x-x_{j+1}\right), \quad \widetilde{e}(x):=\frac{A(x)}{\widetilde{B}(x)} \quad \text { and } \quad \widetilde{B}(x):=\psi(x) B(x) .
$$

Now, by the Leibniz rule,

$$
\begin{aligned}
e^{\prime \prime}(x)= & \sum_{i=0}^{2}\left(\begin{array}{l}
2 \\
i
\end{array}\right) \psi^{(2-i)}(x) \widetilde{e}^{(i)}(x) \\
= & 2 \frac{A(x)}{\widetilde{B}(x)}+2 \psi^{\prime}(x)\left(\frac{A^{\prime}(x)}{\widetilde{B}(x)}-A(x) \frac{\widetilde{B}^{\prime}(x)}{\widetilde{B}^{2}(x)}\right) \\
& +\psi(x)\left(\frac{A^{\prime \prime}(x)}{\widetilde{B}(x)}-2 A^{\prime}(x) \frac{\widetilde{B}^{\prime}(x)}{\widetilde{B}^{2}(x)}+2 A(x) \frac{\widetilde{B}^{\prime 2}(x)}{\widetilde{B}^{3}(x)}-A(x) \frac{\widetilde{B}^{\prime \prime}(x)}{\widetilde{B}^{2}(x)}\right) .
\end{aligned}
$$

Every factor $A^{(k)}(x)$ can be bounded using Lemma 2. In the coming arguments we use the following result. 
Lemma 3 If $d \geq 1$ and if $x \in[a, b]$, then

$$
\frac{1}{|\widetilde{B}(x)|} \leq C h^{d-1}
$$

Proof. For $x \in\left(x_{j}, x_{j+1}\right)$, the definition of $\widetilde{B}$ reads

$$
\widetilde{B}(x)=\psi(x) B(x)=\psi(x) \sum_{i=0}^{n-d} \lambda_{i}(x) .
$$

Since

$$
|B(x)| \geq\left|\lambda_{i}(x)\right|
$$

for any $i \in J_{2}$, we deduce from the definition of $\lambda_{i}$ that

$$
\frac{1}{|\widetilde{B}(x)|} \leq \frac{\prod_{k=i}^{i+d} d_{k}}{d_{j} d_{j+1}}=\prod_{\substack{k=i \\ k \neq j, j+1}}^{i+d} d_{k} \leq C h^{d-1}, \quad \forall i \in J_{2},
$$

which evidently holds also at the nodes.

The factors which remain to be bounded are the following:

$$
N_{1}(x):=\frac{\widetilde{B}^{\prime}(x)}{\widetilde{B}^{2}(x)}, \quad N_{2}(x):=\psi(x) \frac{\widetilde{B}^{\prime}(x)}{\widetilde{B}(x)}, \quad N_{3}(x):=\psi(x) \frac{\widetilde{B}^{\prime \prime}(x)}{\widetilde{B}^{2}(x)} .
$$

We split $\widetilde{B}$ into five parts:

$$
\begin{aligned}
\widetilde{B}(x) & =\psi(x)\left(\sum_{i=0}^{j-d-1} \lambda_{i}(x)+\lambda_{j-d}(x)+\sum_{i=j-d+1}^{j} \lambda_{i}(x)+\lambda_{j+1}(x)+\sum_{i=j+2}^{n-d} \lambda_{i}(x)\right) \\
& =: K_{1}(x)+K_{2}(x)+K_{3}(x)+K_{4}(x)+K_{5}(x) .
\end{aligned}
$$

For symmetry reasons, it is sufficient to study the first three terms, $K_{1}, K_{2}$ and $K_{3}$, since $K_{4}$ and $K_{5}$ are analogous to $K_{2}$ and $K_{1}$. We begin with the first derivative of $K_{1}$ :

$$
K_{1}^{\prime}(x)=\psi^{\prime}(x) \sum_{i=0}^{j-d-1} \lambda_{i}(x)+\psi(x) \sum_{i=0}^{j-d-1} \lambda_{i}^{\prime}(x) .
$$

The terms in both sums alternate in sign and increase in absolute value; we deduce that

$$
\left|K_{1}^{\prime}(x)\right| \leq 2 h \prod_{k=j-d-1}^{j-1} d_{k}^{-1}+d_{j} d_{j+1} \sum_{m=j-d-1}^{j-1} d_{m}^{-1} \prod_{k=j-d-1}^{j-1} d_{k}^{-1} .
$$


We next turn to $K_{2}$, which after simplification reads

$$
K_{2}(x)=\left(x-x_{j+1}\right)(-1)^{j-d} \prod_{k=j-d}^{j-1}\left(x-x_{k}\right)^{-1} .
$$

It follows that

$$
\left|K_{2}^{\prime}(x)\right| \leq \prod_{k=j-d}^{j-1} d_{k}^{-1}+d_{j+1} \sum_{m=j-d}^{j-1} d_{m}^{-1} \prod_{k=j-d}^{j-1} d_{k}^{-1} .
$$

We may rewrite $K_{3}$ as

$$
K_{3}(x)=\sum_{i=j-d+1}^{j}(-1)^{i} \prod_{\substack{k=i \\ k \neq j, j+1}}^{i+d}\left(x-x_{k}\right)^{-1}
$$

which yields the following bound for its derivative:

$$
\left|K_{3}^{\prime}(x)\right| \leq \sum_{i=j-d+1}^{j} \sum_{\substack{m=i \\ m \neq j, j+1}}^{i+d} d_{m}^{-1} \prod_{\substack{k=i \\ k \neq j, j+1}}^{i+d} d_{k}^{-1} .
$$

In view of deriving a bound on $N_{1}$, we first take the quotient of $(32)$ with $|\widetilde{B}(x)|^{2}$. Choosing $i=j-d+1$ in (28), we obtain

$$
\begin{aligned}
\frac{\left|K_{1}^{\prime}(x)\right|}{|\widetilde{B}(x)|^{2}} & \leq 2 h \frac{\prod_{k=j-d+1}^{j-1} d_{k}^{2}}{\prod_{k=j-d-1}^{j-1} d_{k}}+d_{j+1} \sum_{m=j-d-1}^{j-1} \frac{d_{j} \prod_{k=j-d+1}^{j-1} d_{k}^{2}}{d_{m} \prod_{k=j-d-1}^{j-1} d_{k}} \\
& \leq 2 h \frac{\prod_{k=j-d+1}^{j-1} d_{k}}{d_{j-d-1} d_{j-d}}+h \sum_{m=j-d-1}^{j-1} \frac{d_{j} \prod_{k=j-d+1}^{j-1} d_{k}}{d_{m} d_{j-d-1} d_{j-d}} .
\end{aligned}
$$

Since $d_{j-d+1} / d_{j-d-1} \leq 1$ and $d_{j-d+2} / d_{j-d} \leq 1$, and $d_{j} / d_{m} \leq 1$ for $m \leq j-1$, we see that

$$
\frac{\left|K_{1}^{\prime}(x)\right|}{|\widetilde{B}(x)|^{2}} \leq 2 h \prod_{k=j-d+3}^{j-1} d_{k}+h \sum_{m=j-d-1}^{j-1} \prod_{k=j-d+3}^{j-1} d_{k} \leq C h^{d-2} .
$$

Using similar arguments, a bound of the same order may be derived for $\left|K_{2}^{\prime}\right| /|\widetilde{B}|^{2}$ and for $\left|K_{3}^{\prime}\right| /|\widetilde{B}|^{2}$. The result is

$$
\left|N_{1}(x)\right| \leq C h^{d-2} .
$$

To deal with $N_{2}$, we use the mesh ratio $\beta$. Again we begin with the term involving $K_{1}^{\prime}$, choose $i=j-d+1$ in (28) and, instead of cancelling factors in the numerator and denominator, we use the fact that $d_{k} / d_{k-1} \leq 1$ for $k=j-d+1, \ldots, j-1$ :

$$
\begin{aligned}
|\psi(x)| \frac{\left|K_{1}^{\prime}(x)\right|}{|\widetilde{B}(x)|} & \leq 2 h \frac{d_{j} d_{j+1} \prod_{k=j-d+1}^{j-1} d_{k}}{\prod_{k=j-d-1}^{j-1} d_{k}}+d_{j+1}^{2} \sum_{m=j-d-1}^{j-1} \frac{d_{j}^{2} \prod_{k=j-d+1}^{j-1} d_{k}}{d_{m} \prod_{k=j-d-1}^{j-1} d_{k}} \\
& \leq 2 h \frac{d_{j} d_{j+1}}{d_{j-d-1} d_{j-1}}+h \sum_{m=j-d-1}^{j-1} \frac{d_{j}^{2} d_{j+1}}{d_{m} d_{j-d-1} d_{j-1}} .
\end{aligned}
$$


Since $d_{j} / d_{j-d-1} \leq 1$, and $d_{j} / d_{m} \leq 1$ for $m \leq j-1$, we obtain

$$
|\psi(x)| \frac{\left|K_{1}^{\prime}(x)\right|}{|\widetilde{B}(x)|} \leq 2 h \frac{d_{j+1}}{d_{j-1}}+h \sum_{m=j-d-1}^{j-1} \frac{d_{j+1}}{d_{j-1}} \leq C \beta h .
$$

Similar arguments lead to a bound of the same order for $|\psi(x)|\left|K_{2}^{\prime}\right| /|\widetilde{B}|$. For $|\psi(x)|\left|K_{3}^{\prime}\right| /|\widetilde{B}|$ we may cancel the whole product in every term of the inner sum without making use of the mesh ratio:

$$
|\psi(x)| \frac{\left|K_{3}^{\prime}(x)\right|}{|\widetilde{B}(x)|} \leq d_{j} d_{j+1} \sum_{i=j-d+1}^{j} \sum_{\substack{m=i \\ m \neq j, j+1}}^{i+d} d_{m}^{-1} \leq C h .
$$

Thus we have

$$
\left|N_{2}(x)\right| \leq C(\beta+1) h .
$$

A bound for $N_{3}$ may be derived using similar arguments as for $N_{1}$ and the following observation: the differentiation of $\widetilde{B}^{\prime}$ leads to a supplementary factor $\left(x-x_{i}\right)^{-1}$ in some of the terms of $\widetilde{B}^{\prime \prime}$. Since $i \neq j, j+1$, the absolute value of this factor can be eliminated through multiplication with $|\psi|$ :

$$
\frac{|\psi(x)|}{\left|x-x_{i}\right|}=\frac{d_{j} d_{j+1}}{d_{i}} \leq C h
$$

Consequently

$$
\left|N_{3}(x)\right| \leq C h^{d-1}
$$

This last step concludes the proof, since bringing together all the bounds on the terms of the expansion (27) of $e^{\prime \prime}$ yields the claimed result.

Theorem 6 If $d=2$ and if $f \in C^{6}[a, b]$, then

$$
\left\|e^{\prime \prime}\right\| \leq C\left(\beta^{2}+\beta+1\right) h .
$$

Proof. If we expand again the factors $N_{1}, N_{2}$ and $N_{3}$ in (29) in the special case $d=2$, we see that everyone of them may be bounded by a linear function of $\beta$.

\section{Numerical examples}

In order to evaluate the derivatives of $r$, we write it in its barycentric form

$$
r(x)=\frac{\sum_{i=0}^{n} \frac{w_{i}}{x-x_{i}} f_{i}}{\sum_{i=0}^{n} \frac{w_{i}}{x-x_{i}}},
$$

where the formulas for the weights $w_{i}$ are given in [7]. Elegant formulas for the derivatives of an interpolant given in this form have been derived by Schneider and Werner in [11]. 
In particular, using their formulas for the nodal case, one can easily compute the first and second derivatives of $r$ at all the nodes at once. Following [3], it is sufficient to construct differentiation matrices $D^{(1)}$ and $D^{(2)}$ of size $(n+1) \times(n+1)$. If $\mathbf{f}$ is the vector of length $n+1$ of the values $f\left(x_{k}\right)$, then the product $D^{(1)} \mathbf{f}$, respectively $D^{(2)} \mathbf{f}$, yields the vector of the first, respectively second, derivative of $r$ at the nodes.

Table 1: Error in the derivatives of $r$ interpolating $f_{1}$

\begin{tabular}{rcccc}
\hline & \multicolumn{2}{c}{ first derivative } & \multicolumn{2}{c}{ second derivative } \\
\hline$n$ & error & order & error & order \\
\hline 10 & $1.2 \mathrm{e}-01$ & & $5.0 \mathrm{e}-01$ & \\
20 & $5.2 \mathrm{e}-03$ & 4.5 & $4.5 \mathrm{e}-02$ & 3.5 \\
40 & $1.9 \mathrm{e}-04$ & 4.7 & $3.3 \mathrm{e}-03$ & 3.8 \\
80 & $7.2 \mathrm{e}-06$ & 4.7 & $2.5 \mathrm{e}-04$ & 3.7 \\
160 & $2.9 \mathrm{e}-07$ & 4.6 & $2.1 \mathrm{e}-05$ & 3.6 \\
320 & $1.3 \mathrm{e}-08$ & 4.5 & $1.9 \mathrm{e}-06$ & 3.4 \\
640 & $6.8 \mathrm{e}-10$ & 4.3 & $1.9 \mathrm{e}-07$ & 3.3 \\
\hline
\end{tabular}

To illustrate our theoretical results, we started with an example of [7], namely the interpolation of the function $f_{1}(x):=\sin (x)$ for $x \in[-5,5]$. We used the rational interpolant with $d=4$ and sampled $f_{1}$ at equidistant nodes. Our aim was to survey the estimated approximation orders of the derivatives of the interpolant and compare them with the results obtained for the interpolant itself. We computed the error at the same eleven nodes for different values of $n$. Table 1 shows the errors and orders for the first and second derivatives. Comparing these results with the approximated orders in [7], we see that the order decreases almost exactly by one unit at every differentiation.

Table 2: Error in the derivatives of $r$ interpolating $f_{2}$

\begin{tabular}{ccccc}
\hline & \multicolumn{2}{c}{ first derivative } & \multicolumn{2}{c}{ second derivative } \\
\hline$n$ & error & order & error & order \\
\hline 10 & $4.1 \mathrm{e}-01$ & & $1.5 \mathrm{e}+00$ \\
20 & $3.3 \mathrm{e}-02$ & 3.6 & $2.7 \mathrm{e}-01$ & 2.5 \\
40 & $9.4 \mathrm{e}-05$ & 8.5 & $1.6 \mathrm{e}-03$ & 7.4 \\
80 & $1.9 \mathrm{e}-06$ & 5.7 & $7.2 \mathrm{e}-05$ & 4.5 \\
160 & $1.4 \mathrm{e}-07$ & 3.7 & $1.4 \mathrm{e}-05$ & 2.3 \\
320 & $1.2 \mathrm{e}-08$ & 3.5 & $2.3 \mathrm{e}-06$ & 2.7 \\
640 & $1.5 \mathrm{e}-09$ & 3.0 & $3.1 \mathrm{e}-07$ & 2.9 \\
\hline
\end{tabular}

With the next example we studied the convergence rates at intermediate points. For that purpose, we sampled Runge's function $f_{2}(x):=1 /\left(1+x^{2}\right)$ at equidistant nodes in the 
interval $[-5,5]$. We chose $d=3$ and computed the maximum error at 1000 equidistant points inside the interval which are not nodes. Table 2 displays our results, which illustrate Theorems 3 and 5 in this particular case.

Table 3: Error in the derivatives of $r$ interpolating $f_{3}$

\begin{tabular}{rcccc}
\hline & \multicolumn{2}{c}{ first derivative } & \multicolumn{2}{c}{ second derivative } \\
\hline$n$ & error & order & error & order \\
\hline 10 & $2.8 \mathrm{e}-01$ & & $2.0 \mathrm{e}+01$ & \\
20 & $7.7 \mathrm{e}-02$ & 1.9 & $2.0 \mathrm{e}+00$ & 3.3 \\
40 & $1.2 \mathrm{e}-02$ & 2.7 & $5.9 \mathrm{e}-01$ & 1.7 \\
80 & $1.5 \mathrm{e}-03$ & 3.0 & $1.6 \mathrm{e}-01$ & 1.9 \\
160 & $2.0 \mathrm{e}-04$ & 2.9 & $3.9 \mathrm{e}-02$ & 2.0 \\
320 & $2.4 \mathrm{e}-05$ & 3.0 & $9.9 \mathrm{e}-03$ & 2.0 \\
640 & $3.0 \mathrm{e}-06$ & 3.0 & $2.5 \mathrm{e}-03$ & 2.0 \\
\hline
\end{tabular}

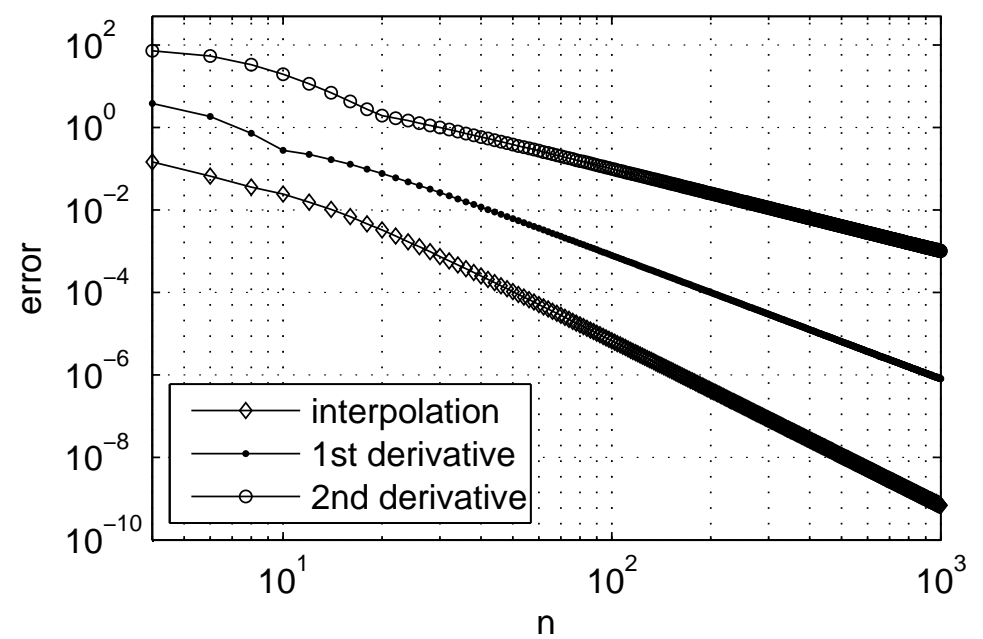

Figure 1: Errors in the rational interpolation with $d=3$ of $f_{3}$ sampled at Chebyshev points in $[-1,1]$ and approximation of its first and second derivatives

As mentioned in the Introduction, we plan to apply the results explained in the present paper to the study of the numerical solution of differential equations. For this reason we experimented with the exact solution of a model problem from Stoer and Bulirsch [12], adapted to the interval $[-1,1]$, namely

$$
f_{3}(x):=\frac{e^{-20}}{1+e^{-20}} e^{10(x+1)}+\frac{1}{1+e^{-20}} e^{-10(x+1)}-\cos ^{2}\left(\frac{\pi}{2}(x+1)\right) .
$$


This time, we sampled the function at Chebyshev points of the second kind and interpolated the computed values using the rational interpolant with $d=3$. Table 3 shows the maximum error at 1000 equidistant points and the experimental convergence rates. It can be proven that for such Chebyshev points of the second kind, the mesh ratio $\beta$ is bounded by 3 for all $n$. Again, the $k$-th derivative of the rational interpolant converges at the rate of $O\left(h^{d+1-k}\right)$ as $h \rightarrow 0$ in the cases $k=1,2$. We also supply a graphical survey of this same experiment at even values of $n$ in Figure 1. In a log-log scale, the errors in the approximation of the first two derivatives of $f_{3}$ are added to those of its rational interpolant. For $n \geq 20$ the curves are nearly straight lines of slopes $-4,-3$ and -2 .

Table 4: Error in the derivatives of the cubic spline interpolating $f_{2}$

\begin{tabular}{rcccc}
\hline & \multicolumn{2}{c}{ first derivative } & \multicolumn{2}{c}{ second derivative } \\
\hline$n$ & error & order & error & order \\
\hline 10 & $7.6 \mathrm{e}-02$ & & $3.7 \mathrm{e}-01$ \\
20 & $2.0 \mathrm{e}-02$ & 1.9 & $2.9 \mathrm{e}-01$ & 0.3 \\
40 & $3.4 \mathrm{e}-03$ & 2.5 & $1.1 \mathrm{e}-01$ & 1.4 \\
80 & $3.9 \mathrm{e}-04$ & 3.1 & $2.5 \mathrm{e}-02$ & 2.2 \\
160 & $4.7 \mathrm{e}-05$ & 3.0 & $6.1 \mathrm{e}-03$ & 2.0 \\
320 & $5.9 \mathrm{e}-06$ & 3.0 & $1.5 \mathrm{e}-03$ & 2.0 \\
640 & $7.1 \mathrm{e}-07$ & 3.1 & $3.8 \mathrm{e}-04$ & 2.0 \\
\hline
\end{tabular}

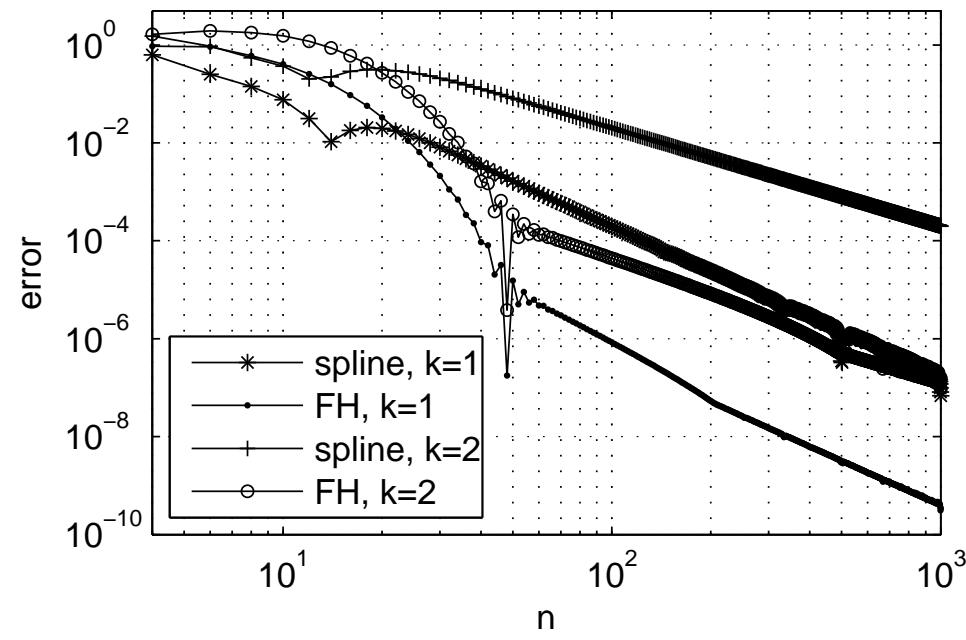

Figure 2: Errors in the spline and rational $(\mathrm{FH})$ approximations with $d=3$ of the first $(k=1)$ and second $(k=2)$ derivatives of $f_{2}$ sampled at equidistant points in $[-5,5]$

We sampled all three functions at equidistant nodes and at Chebyshev points. The 
experimental convergence rates, in the cases not displayed in Tables 1, 2 and 3, are very similar and thus omitted.

Finally we repeated the computation with $f_{2}$, this time using the cubic spline with the not-a-knot end conditions. Since Runge's function is analytic, the spline interpolant and its derivatives have the same convergence orders as the rational interpolant with $d=3$ and its derivatives (see [6]). Table 4 reveals that the experimental orders coincide for large enough $n$, but the error in the rational function is considerably smaller than that of the spline. The difference is due to the faster error decay of the derivatives of the rational interpolant for small values of $n$. Figure 2 confirms this observation: for $n \geq 50$ the curves corresponding to the errors in the spline and rational approximations of the first respectively second derivative of $f_{2}$ are almost parallel.

\section{Conclusion}

Our results show the $O\left(h^{d+1-k}\right)$ convergence of the $k$-th derivative of a family of barycentric rational interpolants for $k=1$ and 2 . The question of a recursive approach for larger $k$ 's arises naturally; we tried to find such a proof, unfortunately without success in the general case studied here. However, the first and last authors have recently discovered a proof that works for the error at the nodes and under the restriction that these are equally or quasi-equally distributed [9].

Acknowledgement. The authors thank the referees for their constructive comments.

\section{References}

[1] K. E. Atkinson, An Introduction to Numerical Analysis 2nd ed., Wiley, New York, 1989.

[2] R. Baltensperger, J.-P. Berrut, and G. Klein, The linear rational collocation method for equidistant and arbitrary points, in preparation.

[3] R. Baltensperger, J.-P. Berrut, and B. Noël, Exponential convergence of a linear rational interpolant between transformed Chebyshev points, Math. Comput. 68 (1999), 1109-1120.

[4] J.-P. Berrut and H. D. Mittelmann, Lebesgue constant minimizing linear rational interpolation of continuous functions over the interval, Comput. Math. Appl. 33 (1997), $77-86$.

[5] L. Bos, S. De Marchi, K. Hormann, and G. Klein, On the Lebesgue constant of barycentric rational interpolation at equidistant nodes, in preparation.

[6] C. de Boor, A Practical Guide to Splines, Applied Mathematical Sciences, vol. 27, Springer, New York, 1978. 
[7] M. S. Floater and K. Hormann, Barycentric rational interpolation with no poles and high rates of approximation, Numer. Math. 107 (2007), 315-331.

[8] E. Isaacson and H. B. Keller, Analysis of Numerical Methods, Wiley, New YorkLondon-Sydney, 1966.

[9] G. Klein and J.-P. Berrut, Linear rational finite differences from derivatives of barycentric rational interpolants, submitted.

[10] W. H. Press, S. A. Teukolsky, W. T. Vetterling, and B. P. Flannery, Numerical Recipes: The Art of Scientific Computing 3rd ed., Cambridge University Press, Cambridge, 2007.

[11] C. Schneider and W. Werner, Some new aspects of rational interpolation, Math. Comp. 47 (1986), 285-299.

[12] J. Stoer and R. Bulirsch, Numerische Mathematik II. 3., verb. Aufl., Springer, Berlin, 1990. 